\title{
Ionisation Penning de l'azote moléculaire par He 2 1S et 2 3S
}

\author{
F. Tuffin, G. Le Coz et J. Peresse \\ Laboratoire de Collisions Electroniques et Atomiques $\left({ }^{*}\right)$ \\ Université de Bretagne Occidentale, Faculté des Sciences et Techniques, 6, avenue le Gorgeu, 29283 Brest Cedex, France
}

(Reçu le 11 octobre 1979, révisé le 23 novembre 1979, accepté le 10 janvier 1980)

\begin{abstract}
Résumé. - Les auteurs ont étudié le spectre en énergie des électrons éjectés au cours de l'ionisation Penning de l'azote moléculaire par les métastables de l'hélium. Un modèle, faisant intervenir la formation d'une quasimolécule, est proposé pour rendre compte de certaines anomalies dans l'ionisation Penning par l'état métastable $2{ }^{3} \mathrm{~S}$. Les auteurs suggèrent également la présence d'un état autoionisant de $\mathrm{N}_{2}$ qui aurait pour effet d'accroître la section efficace d'ionisation par l'état métastable $2{ }^{1} \mathrm{~S}$.
\end{abstract}

Abstract. - A study is reported of the ejected electron spectra resulting from Penning ionization of a molecular nitrogen target by impact of helium metastables. A model is proposed for ionization by the $2{ }^{3} \mathrm{~S}$ metastable in which the formation of a quasi-molecular complex is invoked, and it is suggested that the presence of an autoionizing state of $\mathrm{N}_{2}$ leads to enhancement of certain cross sections for ionization by the $2{ }^{1} \mathrm{~S}$ metastable.

1. Introduction. - Dans un article précédent (Tuffin et al. [1]), nous avons discuté l'ionisation Penning d'une cible atomique $\mathrm{Y}$ par la désexcitation d'un métastable $\mathrm{X}$ et nous avons présenté des résultats expérimentaux pour la distribution en énergie des électrons éjectés. Cette distribution en énergie est directement reliée à la différence entre les courbes de potentiel $V^{*}$ pour le système $\mathrm{X}^{*} \mathrm{Y}$ et $V^{+}$pour le système $\mathrm{XY}^{+}$, parce qu'au cours de la transition, l'énergie cinétique relative du projectile et de la cible ne change pas.

Dans le présent article, nous discutons l'ionisation Penning d'une molécule diatomique. Alors que la cible $\mathrm{Y}$ est dans son état fondamental, l'ion moléculaire $\mathrm{Y}_{i, v}^{+}$est formé dans un état excité, $i$ et $v$ représentent respectivement les nombres quantiques électroniques et vibrationnels.

$$
\mathrm{X}^{*}+\mathrm{Y} \rightarrow \mathrm{X}+\mathrm{Y}_{i, v}^{+}+\mathrm{e}^{-}\left(E_{\mathrm{el}}, i, v\right)+\delta\left(E_{\mathrm{c}}, i, v\right)
$$

où $E_{\mathrm{el}}$ est l'énergie de l'électron éjecté et où $\delta\left(E_{\mathrm{c}}, i, v\right)$ est un transfert d'énergie cinétique, $\delta$ étant l'opposé de la quantité $\Delta$ définie dans l'éq. (2) ci-dessous. Dans le cas présent, on ne peut plus considérer des courbes de potentiel, mais on doit travailler en termes de surfaces de potentiel $V^{*}(\mathbf{R})$ et $V_{i, v}^{+}(\mathbf{R})$ où $\mathbf{R}$ est le vecteur position du projectile par rapport au centre

(*) Equipe de Recherche associée au C.N.R.S. $\mathrm{n}^{\circ} 256$. de masse de la molécule et à l'axe moléculaire. Le potentiel $V^{+}$dépend de l'état de l'ion moléculaire créé; les valeurs limites de $V^{*}$ et $V^{+}$pour $\mathbf{R}$ grand étant indépendantes de sa direction :

$$
\begin{gathered}
V^{*}(\mathbf{R})_{|R| \tilde{F} \infty} V^{*}(\infty) \\
V_{i, r}^{+}(\mathbf{R})_{\mid R \tilde{\mid} \rightarrow \infty} V_{i, r}^{+}(\infty) .
\end{gathered}
$$

On peut également définir les énergies cinétiques relatives $E_{\mathrm{c}}(\mathbf{R})$ et $E_{\mathrm{c}}^{i, v}\left(\mathbf{R}, \mathbf{R}_{1}\right)$ pour les voies d'entrée et de sortie, où $E_{\mathbf{c}}^{i, v}$ dépend du vecteur $\mathbf{R}_{1}$, valeur de $R$ lorsque la transition a lieu. Ces quantités ont des valeurs limites $E_{\mathrm{c}}(\infty)$ et $E_{\mathrm{c}}^{i, v}\left(\infty, \mathbf{R}_{1}\right)$ respectivement (voir Fig. 1).

Puisqu'on ne peut pas nécessairement supposer que l'énergie cinétique relative est conservée au moment de la transition (i.e. que $E_{\mathrm{c}}\left(\mathbf{R}_{1}\right)=E_{\mathrm{c}}^{i, v}\left(\mathbf{R}_{1}, \mathbf{R}_{1}\right)$ ), on a :

$$
E_{\mathrm{el}}\left(R_{1}\right)-E_{0}^{i, v}=E_{\mathrm{c}}(\infty)-E_{\mathrm{c}}^{i, v}\left(\infty, \mathbf{R}_{1}\right)=\Delta^{i, v}\left(\mathbf{R}_{1}\right)
$$

où

$$
E_{0}^{i, v}=V^{*}(\infty)-V_{i, v}^{+}(\infty) .
$$

Il n'est pas certain que dans le cas d'une cible moléculaire, l'analyse des résultats expérimentaux au moyen des surfaces de potentiel fournisse des résultats intéressants. Nos résultats expérimentaux montrent une différence entre l'espacement des niveaux vibrationnels de l'état $\mathrm{A}^{2} \Pi_{\mathrm{u}}$ obtenu par photoionisation et par impact de l'hélium métastable dans l'état $2{ }^{3} \mathrm{~S}$. 


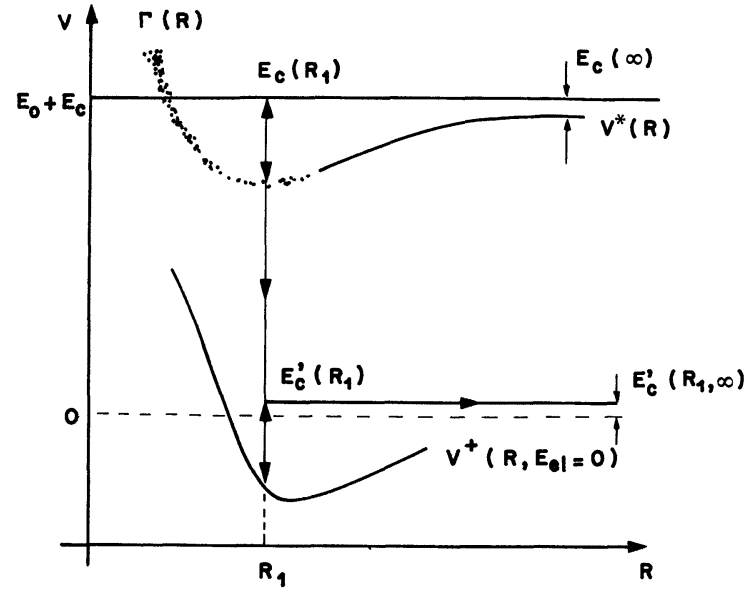

Fig. 1. - Modèle courbes de potentiel pour l'ionisation Penning. $\Gamma(R)$ représente la largeur de la courbe $V^{*}(R)$.

[Potential curve model of Penning ionization.]

Il semble que dans ce cas l'ionisation Penning de $\mathrm{N}_{2}$ procède par l'intermédiaire de la formation d'une quasi-molécule excitée $\mathrm{N}_{2} \mathrm{He}$ de durée de vie assez longue. On note également la présence d'un effet dû à un état autoionisant de $\mathrm{N}_{2}$ situé à $4,66 \mathrm{eV}$ au-dessus du seuil d'ionisation. Ces points seront discutés au paragraphe 3 .

2. Dispositif expérimental. - Le dispositif expérimental a déjà été décrit dans l'article précédent, dans le présent article nous ne donnerons que les aspects essentiels (voir Fig. 2). Le jet d'hélium métastable est produit par bombardement électronique d'un faisceau d'hélium, les électrons ayant une énergie comprise entre 30 et $40 \mathrm{eV}$, l'intensité du faisceau d'électrons étant d'environ $1 \mathrm{~mA}$. Après collimation, le faisceau d'hélium métastable pénètre dans une chambre de collisions de grandes dimensions où il entre en interaction avec le faisceau cible d'azote moléculaire. Les électrons éjectés au cours de l'ionisation Penning

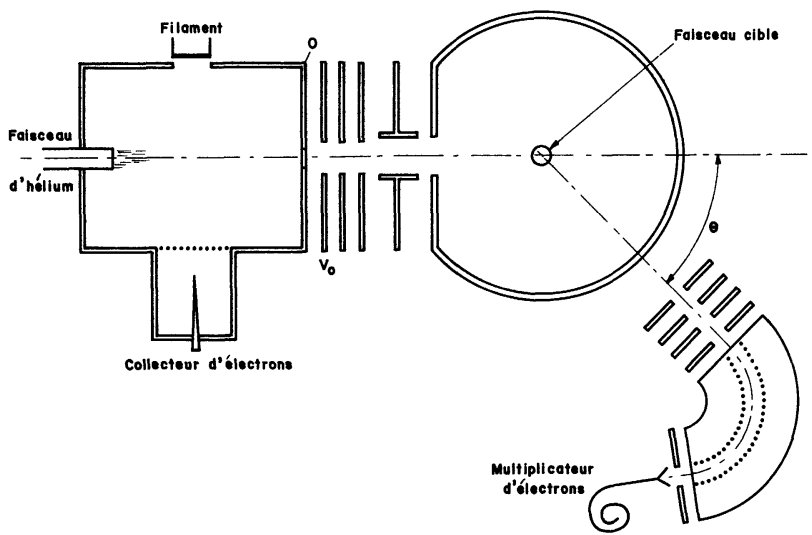

Fig. 2. - Schéma de principe du dispositif expérimental.

[Schematic drawing of the main parts of the apparatus.] sont analysés en énergie au moyen d'un sélecteur électrostatique $127^{\circ}$ et détectés par un channeltron. Le système de détection peut tourner autour du faisceau cible. Les surfaces métalliques sont recouvertes d'or afin de diminuer le bruit de fond.

3. Résultats expérimentaux. - Nous présentons figure 3 un spectre en énergie des électrons éjectés, caractéristique de nos résultats expérimentaux. Ce spectre montre que nous disposons d'un dispositif expérimental fournissant un bon rapport signal sur bruit, une bonne distribution en énergie, un faible bruit de fond, même du côté des électrons d'énergies voisines de zéro, donc une bonne précision dans la détermination des positions des différentes structures. Les trois états électroniques $\mathrm{X}^{2} \Sigma_{\mathrm{g}}^{+}, \mathrm{A}^{2} \Pi_{\mathrm{u}}$ et $\mathrm{B}^{2} \Pi_{\mathrm{u}}^{+}$ sont accessibles par les trois processus d'ionisation suivants : par impact des métastables $2{ }^{3} \mathrm{~S}$ d'une part et $2{ }^{1} \mathrm{~S}$ d'autre part, et par impact des photons de longueur d'onde $584 \AA$ résultant de la désexcitation du niveau $2{ }^{1} \mathrm{P}$ de l'hélium. Chaque état électronique de l'ion $\mathrm{N}_{2}^{+}$possède un ensemble de niveaux vibrationnels; il y a ainsi, en principe, neuf ensembles de structures vibrationnelles. Malheureusement, les différences d'énergie sont telles qu'il y a un recouvrement considérable des différents ensembles de structures. Cependant, compte tenu des conditions expéri-

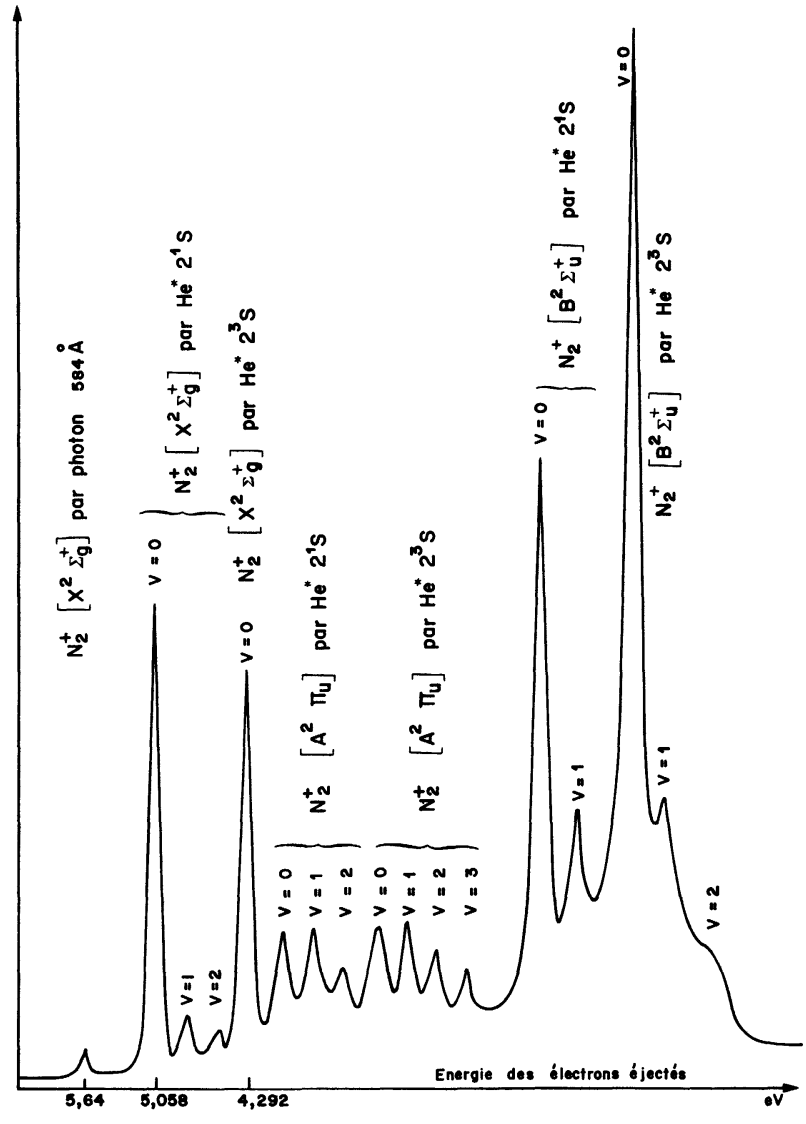

Fig. 3. - Spectre d'énergie des électrons éjectés au cours de l'ionisation Penning de l'azote moléculaire.

[Penning electron spectra of nitrogen.] 


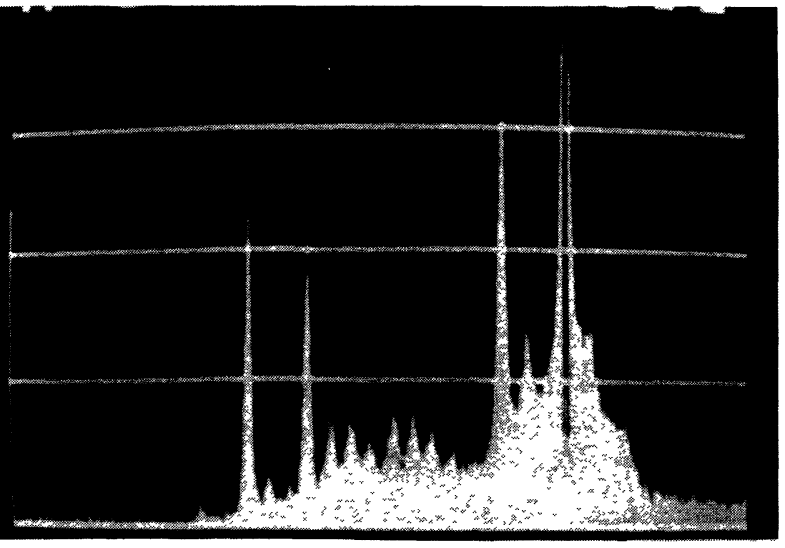

Photo. - Photographie d'un spectre d'énergie des électrons éjectés par ionisation Penning de l'azote moléculaire.

[Photograph of an energy spectrum of electrons ejected in Penning ionization of molecular nitrogen.]

mentales, l'intensité du flux de photons est faible. Sur le spectre de la figure 3 , seul est visible le pic correspondant à la photoionisation sur l'état $\mathrm{X}^{2} \Sigma_{\mathrm{g}}^{+} v=0$. En général, on n'observe que les niveaux vibrationnels avec $v \leqslant 2$, une exception concerne le niveau $v=3$ de l'état $\mathrm{A}^{2} \Pi_{\mathrm{u}}$ créé par impact du métastable $2{ }^{3} \mathrm{~S}$. Notre résolution en énergie est suffisante pour déterminer avec précision la position des différentes structures; le pic correspondant à la photoionisation nous fournit une origine pour l'échelle des énergies. Comme dans le cas où la cible est un gaz rare, les structures dues à l'ionisation Penning sont déplacées par rapport à la position obtenue en faisant simplement la différence entre l'énergie d'excitation du métastable et l'énergie nécessaire pour ioniser la molécule $\mathrm{N}_{2}$ sur un niveau électronique et vibrationnel particulier de $\mathrm{N}_{2}^{+}$. Ces déplacements d'énergie ( $\Delta$ ) semblent être indépendants pour les niveaux vibrationnels $v=0$ de l'état électronique de $\mathrm{N}_{2}^{+}$mais dépendent de l'état du projectile. On obtient
$\Delta=20 \mathrm{meV}$ pour $2{ }^{1} \mathrm{~S}$ et $\Delta=50 \mathrm{meV}$ pour $2{ }^{3} \mathrm{~S}$, en bon accord avec les résultats de Hotop et al. [2] et de Yee et al. [3]. Ces deux expériences utilisent une source d'hélium métastable du même type que celle que nous avons adoptée. Alors que nous utilisons un sélecteur électrostatique $127^{\circ}$ pour étudier l'énergie des électrons éjectés, la technique utilisée par Hotop et al. est du type champ retardateur. Nous étudions les électrons éjectés sous un angle de $80^{\circ}$ par rapport à la direction du faisceau de métastables, alors que les expériences des autres auteurs sont effectuées à $90^{\circ}$.

Toutefois, nous ne pensons pas que ces quelques différences de technique puissent conduire à des différences significatives de résultats.

Il est par conséquent quelque peu surprenant de constater que des différences importantes, en ce qui concerne l'espacement des niveaux vibrationnels par rapport aux résultats de ces différents auteurs, sont mises en évidence. Dans les tableaux I et II nous comparons nos résultats en ce qui concerne l'espacement des niveaux vibrationnels et leur intensité avec les résultats obtenus par ionisation Penning et par photoionisation. Ces résultats sont la moyenne d'un grand nombre de résultats du type de celui présenté figure 3 .

En général, nous mesurons des déviations plus importantes par rapport aux résultats de photoionisation que Hotop et al. et Yee et al. Nos résultats sont particulièrement intéressants pour l'ionisation Penning sur l'état $\mathrm{A}^{2} \Pi_{\mathrm{u}}$ de $\mathrm{N}_{2}^{+}$par impact du métastable $2{ }^{3} \mathrm{~S}$. Dans ce cas, l'écart entre les niveaux vibrationnels $v=2$ et $v=3$ est plus important que l'écart entre les niveaux $v=0$ et $v=1$, ainsi que $v=1$ et $v=2$. Ce fait est évident sur la photo du spectre que nous présentons et se retrouve sur chaque spectre. Nous avons vérifié la linéarité de notre échelle d'énergie. Comme nous l'avons déjà souligné, l'analyse des différentes séries de structures est compliquée du fait de leur interférence, aussi avons-nous soigneu-

Tableau I. - Probabilités de transition vibrationnelle pour $\mathrm{N}_{2}(\mathrm{X} ; v=0) \rightarrow \mathrm{N}_{2}^{+}\left(\mathrm{A}, \mathrm{B}, v^{\prime}\right)$.

[Vibrational transition probability for $\mathrm{N}_{2}(\mathrm{X} ; v=0) \rightarrow \mathrm{N}_{2}^{+}\left(\mathrm{A}, \mathrm{B}, v^{\prime}\right)$.]

J. L. Gardner et al. (photoionisation) [13]

H. Hotop et al. (photoionisation) [2]

D. S. C. Yee et al. [3]

H. Hotop et al. $\mathrm{He} 2{ }^{3} \mathrm{~S}$ [2]

D. S. C. Yee et al. He $2{ }^{3} \mathrm{~S}$ [3]

H. Hotop et al. He $2{ }^{3} \mathrm{~S}$ [14]

Présent travail $\mathrm{He} 2{ }^{3} \mathrm{~S}$

H. Hotop et al. He $2{ }^{1} \mathrm{~S}$ [2]

D. S. C. Yee et al. He $2{ }^{1} \mathrm{~S}$ [3]

Présent travail $\mathrm{He} 2{ }^{1} \mathrm{~S}$
Etat électronique

\begin{tabular}{|c|c|c|c|c|c|c|}
\hline \multicolumn{2}{|c|}{$\mathrm{X}^{2} \Sigma_{\mathrm{g}}^{+}$} & \multicolumn{5}{|c|}{$\mathrm{A}^{2} \Pi_{\mathrm{u}}$} \\
\hline 1 & 2 & 0 & 1 & 2 & 3 & 4 \\
\hline - & - & - & - & - & - & - \\
\hline 100 & & 87 & 100 & 76 & 44 & 19 \\
\hline 100 & & 86 & 100 & 73 & 46 & 21 \\
\hline 100 & & 87 & 100 & 70 & 40 & 16 \\
\hline 100 & & 89 & 100 & 65 & 35 & 17 \\
\hline 100 & & 86 & 100 & 63 & 30 & \\
\hline 100 & & 89 & 100 & 67 & 34 & 13 \\
\hline 100 & & 88 & 100 & 77 & 40 & \\
\hline 100 & & & & & & \\
\hline 100 & & & 100 & 57 & & \\
\hline 100 & 4 & 87 & 100 & 56 & & \\
\hline
\end{tabular}

\begin{tabular}{lll}
\multicolumn{3}{c}{$\mathrm{B}^{2} \Sigma_{\mathrm{u}}^{+}$} \\
0 & 1 & 2 \\
- & - & - \\
100 & 10 & \\
100 & 14 & \\
100 & 15 & \\
100 & 18 & \\
100 & 16 & \\
100 & 21 & 8 \\
100 & 20 &
\end{tabular}


Tableau II. - Espacement entre les différents niveaux vibrationnels de $\mathrm{N}_{2}^{+}$; comparaison avec les résultats de photoionisation.

[Vibrational level splittings of $\mathrm{N}_{2}^{+}$; comparison with photoionization results.]

\begin{tabular}{|c|c|c|c|c|c|c|c|c|}
\hline \multirow[b]{2}{*}{ Auteurs } & & \multicolumn{2}{|c|}{$\mathrm{X}^{2} \Sigma_{\mathrm{u}}^{+}$} & \multicolumn{3}{|c|}{ Etat de l'ion $\mathrm{N}_{2}^{+}$} & \multicolumn{2}{|r|}{$\mathrm{B}^{2} \Sigma_{\mathrm{u}}^{+}$} \\
\hline & Méthode & $0-1$ & $1-2$ & $0-1$ & $1-2$ & $2-3$ & $3-4$ & $0-1$ \\
\hline - & - & - & - & - & - & - & - & - \\
\hline Turner et al. [9] & Photoionisation & 290 & - & 230 & 230 & 220 & 200 & 270 \\
\hline Frost et al. [10] & - & 330 & - & 230 & 230 & 220 & 210 & - \\
\hline Spohr et al. [11] & - & 300 & 300 & 220 & 220 & 220 & 220 & 160 \\
\hline Collin et al. [12] & - & 271 & 259 & 235 & 226 & 225 & 211 & 304 \\
\hline Hotop et al. [2] & Penning He $2{ }^{1} \mathrm{~S}$ & 280 & - & - & - & - & - & 285 \\
\hline Présent travail & - & 269 & 260 & 252 & 234 & - & - & 304 \\
\hline Hotop et al. [2] & Penning He $2{ }^{3} \mathrm{~S}$ & $275 \pm 10$ & - & $230 \pm 10$ & $225 \pm 10$ & $225 \pm 15$ & $215 \pm 15$ & 290 \\
\hline Présent travail & - & - & - & $243 \pm 6$ & $243 \pm 6$ & $248 \pm 6$ & - & 280 \\
\hline
\end{tabular}

sement examiné une éventuelle perturbation de ce pic par des structures dues à l'ionisation sur l'état $\mathrm{A}^{2} \Pi_{\mathrm{u}}$ par impact du métastable $2{ }^{1} \mathrm{~S}$ ou par photoionisation sur l'état $\mathrm{B}^{2} \Sigma_{\mathrm{u}}^{+}$. Aucun de ces processus ne peut perturber de manière significative le pic correspondant à $\mathrm{A}^{2} \Pi_{\mathrm{u}} v=3$. En analysant nos résultats, nous avons supposé que les populations relatives des différents niveaux vibrationnels de l'état électronique $\mathrm{X}^{2} \Sigma_{\mathrm{g}}^{+}$sont essentiellement indépendantes de l'état du projectile $\left(2{ }^{1} \mathrm{~S}\right.$ ou $\left.2{ }^{3} \mathrm{~S}\right)$.

On constate sur la figure 3 que la série

$$
\mathrm{He}\left(2{ }^{1} \mathbf{S}\right) / \mathrm{N}_{2}^{+}\left(\mathrm{A}^{2} \Pi_{\mathrm{u}}\right)
$$

interfère avec la série $\mathrm{He}\left(2^{3} \mathrm{~S}\right) / \mathrm{N}_{2}^{+}\left(\mathrm{X}^{2} \Sigma_{\mathrm{g}}^{+}\right)$. Pour avoir une estimation des populations relatives des niveaux vibrationnels de l'état $\mathrm{A}^{2} \Pi_{\mathrm{u}}$ créé par $\mathrm{He}^{*}\left(2{ }^{1} \mathrm{~S}\right)$, nous procédons de la manière suivante : nous avons obtenu les populations relatives de la série $\mathrm{He}^{*}\left(2{ }^{1} \mathrm{~S}\right) / \mathrm{N}_{2}^{+}\left(\mathrm{X}^{2} \Sigma_{\mathrm{g}}^{+}\right)$, à savoir $100,9,4$, nous en déduisons, sur la base de l'hypothèse énoncée précédemment, l'importance de la contribution dans les niveaux $v=0$ et $v=1$ de l'état $\mathrm{A}^{2} \Pi_{\mathrm{u}}$ des niveaux $v=1$ et $v=2$ du niveau $\mathrm{X}^{2} \Sigma_{\mathrm{g}}^{+}$créé par $\mathrm{He}^{*}\left({ }^{3} \mathrm{~S}\right)$, nous obtenons ainsi des populations relatives de 87 , 100 et 56 respectivement pour les niveaux vibrationnels $v=0,1$ et 2 . Il est évident qu'un tel procédé n'a de signification que parce que la population des niveaux $v=1$ et $v=2$ de l'état $\mathrm{X}^{2} \Sigma_{\mathrm{g}}^{+}$est faible par rapport au niveau $v=0$.

On remarquera de la même manière que les deux séries $\mathrm{He}\left(2^{3} \mathrm{~S}\right) / \mathrm{N}_{2}^{+}\left(\mathrm{A}^{2} \Pi_{\mathrm{u}}\right)$ et $\mathrm{He}\left({ }^{1} \mathrm{~S}\right) / \mathrm{N}_{2}^{+}\left(\mathrm{A}^{2} \Pi_{\mathrm{u}}\right)$ interfèrent. Il est en particulier nécessaire d'estimer la contribution du niveau $\mathrm{He}\left(2{ }^{1} \mathrm{~S}\right) / \mathrm{N}_{2}^{+}\left(\mathrm{A}^{2} \Pi_{\mathrm{u}} v=3\right)$ au niveau $\mathrm{He}\left(2^{3} \mathrm{~S}\right) / \mathrm{N}_{2}^{+}\left(\mathrm{A}^{2} \Pi_{\mathrm{u}} v=0\right)$. Pour effectuer cette estimation, on tient compte du fait que Hotop et al., dans leurs conditions expérimentales, n'ont pas observé de différences significatives entre les populations de l'état $\mathrm{N}_{2}^{+}\left(\mathrm{A}^{2} \Pi_{\mathrm{u}}\right)$ créé par $\mathrm{He}^{*}{ }^{3}{ }^{3} \mathrm{~S}$ et par photoionisation. Ces dernières étant bien connues, on retranche du niveau $v=0$ la contribution ainsi estimée du niveau $v=4$ créé par $\operatorname{He}^{*}\left(2{ }^{1} S\right)$. On constate que la perturbation des niveaux $v=2$ et $v=3$ de l'état $A_{\perp}^{L} \Pi_{\mathrm{u}}$ créé par $\mathrm{He}^{*}\left(2{ }^{3} \mathrm{~S}\right)$ est très faible et ne peut expliquer l'anomalie observée dans l'écart entre les niveaux vibrationnels $v=2$ et $v=3$. Une telle modification de l'écart entre ces niveaux vibrationnels par rapport à l'écart observé par photoionisation, indique clairement une modification de la courbe de potentiel de l'ion moléculaire. Comme en général le bas d'un puits de potentiel est moins aisément perturbé que le haut, on doit observer une modification de l'écart entre les niveaux vibrationnels plus importants pour les grandes valeurs de $v$ que pour les valeurs les plus basses. C'est effectivement ce que l'on observe.

Notre résultat semble suggérer le modèle suivant pour l'ionisation Penning par le métastable $2{ }^{3} \mathrm{~S}$. La collision s'effectue par l'intermédiaire de la formation d'une molécule complexe excitée. Quand la distance projectile-cible est suffisamment faible, un électron de la molécule d'azote va remplir la couche incomplète $1 \mathrm{~s}$ de l'hélium métastable, alors que l'électron $2 \mathrm{~s}$ de l'hélium se place sur un niveau hautement excité et autoionisant de la molécule $\left(\mathrm{HeN}_{2}\right)$. Ce niveau autoionisant qui se dissocie dans les produits finaux de la réaction, à savoir l'hélium dans l'état fondamental et la molécule d'azote ionisée dans différents niveaux électroniques vibrationnellement excités, doit avoir une durée de vie assez longue de telle sorte que les interactions soient en mesure de perturber le mouvement des deux noyaux $\mathrm{N}-\mathrm{N}$ entraînant un déplacement des niveaux de vibration. Les expériences de I. V. Hertel et al. $[15,16]$ sur le quenching de $\mathrm{Na}^{*}$ par $\mathrm{N}_{2}$ semblent montrer une telle déformation de la molécule $\mathrm{N}_{2}$. Ce modèle conforme au fait que l'ionisation Penning s'effectue par l'intermédiaire d'un processus d'échange, permet également, en supposant l'existence du croisement d'un grand nombre de niveaux autoionisants de la molécule $\left(\mathrm{HeN}_{2}\right)$, d'expliquer l'existence d'un fond continu sur lequel se greffent les structures dans le cas de l'ionisation Penning des molécules [17]. Une telle perturbation des niveaux vibrationnels implique qu'il doit y avoir une forte dépendance de l'anomalie observée avec l'énergie 
cinétique relative du projectile et de la cible et également avec l'angle d'excitation de l'électron. La perturbation devant être favorisée aux courtes distances projectiles-cibles et devant donc croître avec l'énergie de collision.

Des différences importantes par rapport aux facteurs de Franck-Condon ont été observées par Cermak $[4,5]$ pour l'ionisation Penning conduisant à la formation de $\mathrm{O}_{2}^{+}\left(\mathrm{A}^{2} \Pi_{\mathrm{u}}\right), \mathrm{HBr}^{+}\left(\mathrm{A}^{2} \Sigma^{+}\right)$et $\mathrm{HCl}^{+}\left(\mathrm{A}^{2} \Sigma^{+}\right)$. Cermak [6] a également observé, dans un certain nombre de réactions, des différences importantes par rapport à la photoionisation, dans les populations relatives des différents états électroniques. Cette dernière observation a été confirmée par Hotop et al. et par Yee et al. Nous aboutissons à la même conclusion. Le spectre de la figure 3 montre que l'état $\mathrm{B}^{2} \Sigma_{\mathrm{u}}^{+}$est plus peuplé que $\mathrm{X}^{2} \Sigma_{\mathrm{g}}^{+}$, contrairement aux observations faites par photoionisation.

Dans un travail précédent sur l'ionisation de $\mathrm{N}_{2}$ par impact électronique, Tuffin [7] a montré l'existence d'une résonance pour une énergie de 4,65 eV au-dessus du seuil d'ionisation, résonance également observée par Weissler [8] par impact de photons. Cette résonance correspond à un état autoionisant d'énergie d'excitation de $20,24 \mathrm{eV}$ proche de l'état métastable $2{ }^{1} \mathrm{~S}$. Il y a donc une possibilité pour que l'ionisation Penning par l'état $2{ }^{1} \mathrm{~S}$ soit influencée par la formation d'un état intermédiaire autoionisant se désexcitant pour donner un ion dans l'état $\mathrm{B}^{2} \Sigma_{\mathrm{u}}^{+}$.
4. Discussion. - Les résultats que nous avons présentés ci-dessus montrent qu'il existe un certain nombre de différences entre nos résultats et ceux de H. Hotop et al. Les expériences de Hotop et al. furent réalisées à l'aide de la technique du potentiel retardé pour la mesure de la distribution en énergie des électrons éjectés, ces électrons étant collectés à $90^{\circ}$. On peut noter que cette technique implique une dérivation de la courbe expérimentale obtenue, ce qui entraîne une moins bonne définition des structures surtout lorsque celles-ci sont faibles comme c'est le cas des niveaux vibrationnels de l'état $A^{2} \Pi_{u}$. On peut remarquer également que le dispositif expérimental utilisé collecte les électrons émis dans diverses directions autour de $90^{\circ}$, ceci augmentant l'intensité du signal mais dissimulant d'éventuels effets angulaires. Notre propre dispositif expérimental évite de telles difficultés.

Les expériences réalisées par $\mathrm{H}$. Hotop le furent pour une énergie de collision de l'ordre de $300 \mathrm{~K}$. Notre propre expérience est réalisée pour une énergie supérieure à $500 \mathrm{~K}$, ceci pouvant permettre d'atteindre de plus courtes distances projectiles-cibles favorisant la création d'un complexe au cours de la collision.

Ces remarques montrent la nécessité d'étudier la distribution en énergie, en fonction de l'énergie cinétique relative des deux partenaires et en fonction de la distribution angulaire de l'électron éjecté pour parfaire le modèle proposé.

\section{Bibliographie}

[1] Tuffin, F., Le Coz, G. and Peresse, J., J. Physique Lett. 40 (1979) L-271.

[2] Hотор, H. and Niehaus, A., Int. J. Mass. Spectrom. Ion Phys. 3 (1970) 415.

[3] Yee, D. S. C., Stewart, W. B., McDowell, C. A. and Brion, C. E., J. Electron Spectrosc. Relat. Phenom. 7 (1975) 93.

[4] Cermak, V., J. Electron Spectrosc. Relat. Phenom. 8 (1976) 325.

[5] Cermak, V., J. Electron Spectrosc. Relat. Phenom. 9 (1976) 419.

[6] Cermak, V., Collect. Czech. Chem. Commun. 33 (1968) 2739.

[7] Tuffin, F., Thèse, Brest (1968).

[8] Weissler, G. L., Handb. Phys. Berlin XXI (1956) 328.

[9] Turner, D. W. and May, D. P., J. Chem. Phys. 46 (1966) 471.
[10] Frost, D. C., McDowell, C. A. and Vroom, D. A., Proc. R. Soc. (London) A 296 (1967) 566.

[11] SpOHR, T. and PUTTKAMER, E. V., Z. Naturforsch. 22a (1967) 705.

[12] Collin, J. E. and Natales, P., Int. J. Mass. Spectrom. Ion Phys. 2 (1969) 231.

[13] Gardner, J. L. and Samson, J. A. R., J. Chem. Phys. 60 (1974) 3711

[14] Нотор, H. and HÜвLER, G., J. Electron Spectrosc. Relat. Phenom. 11 (1977) 101.

[15] Hertel, I. V. and Stoll, W., Adv. Atom. Molec. Phys. (1977) 113.

[16] Hertel, I. V., Hofmann, H. and Rost, K. A., Phys. Rev. Lett. 36 (1976) 861.

[17] Tuffin, F., Le Coz, G. and Peresse, J., Phys. Lett. 73A (1979) 163 